\title{
Investigation of Phase Transformations in Ge4Sb4Te5 film using Transmission Electron Microscopy
}

Manish Singh ${ }^{1}$, Chanchal Ghosh ${ }^{1}$, Paul Kotula ${ }^{2}$, Benjamin Miller ${ }^{3}$, John Watt ${ }^{4}$, Helena Silva ${ }^{1}$ and C. Barry Carter $^{1}$

${ }^{1}$ University of Connecticut, Connecticut, United States, ${ }^{2}$ Sandia National Laboratories, Albuquerque, New Mexico, United States, ${ }^{3}$ Gatan Inc., California, United States, ${ }^{4}$ Los Alamos National Laboratory, Connecticut, United States

Phase-change materials can exhibit a rather unconventional combination of properties. They display a large change in electrical resistivity and optical reflectivity during the phase transformation from amorphous to crystalline state, which suggests a significant change in local atomic configuration. However, transformation from the amorphous to crystalline phase takes place very quickly. This property combination makes these materials suitable for memory applications (Wuttig \& Yamada, 2007). Ge-SbTe (GST)-based phase-change materials have been studied extensively, in particular the stoichiometric $\mathrm{Ge}_{2} \mathrm{Sb}_{2} \mathrm{Te}_{5}$ (GST-225) alloy lying on the GeTe-Sb2 $\mathrm{Te}_{3}$ tie line, owing to its optimum thermal stability of amorphous phase at room temperature and crystallization speed. The GST-225 goes from an amorphous state to a crystalline (face-centered cubic) state upon heating with a typical crystallization temperature of $\sim 170{ }^{\circ} \mathrm{C}$ (Yamada, 2012). The time scale of the transformation in GST-225 has been observed to be on the order of nanoseconds and this rapid change has been ascribed to unchanged chemistry after phase change. However, uncapped GST-225 films exposed to atmosphere for longer durations show crystallization at $\sim 35{ }^{\circ} \mathrm{C}$ along with chemical partitioning in GST-225, which becomes pronounced at higher temperatures (Singh, et al., 2020b; Tripathi, et al., 2020).

The investigation of GST alloys having compositions outside the prototypical $\mathrm{Ge}_{2} \mathrm{Sb}_{2} \mathrm{Te}_{5}$ has also been carried out in quest of improved performance of the GST-based devices. It has been demonstrated that Ge-rich GST is suitable for high temperature applications and high-speed performance can be achieved with Sb-rich GST alloys. One interesting aspect of investigation could be to study GST alloys with both Ge and Sb-rich composition in order to optimize the tradeoff between thermal stability and crystallization speed.

In this investigation a Ge4Sb4Te5 (GST-445) film $25 \mathrm{~nm}$ thick was directly deposited on a Protochips MEMS heating device using magnetron sputtering and kept uncapped specifically for in-situ TEM study (Ghosh, et al., 2020; Singh, et al., 2020a). In-situ heating experiments were carried out in a $\mathrm{C}_{\mathrm{S}}$-corrected FEI Titan ETEM employing an Aduro 300 Protochips holder. Dynamics of crystallization were followed using a large field of view, high-speed camera (Gatan K3 IS) @ 10 frames per second with a low-dose rate $\left(12.7 \mathrm{e}^{-} \AA^{-2} \mathrm{~s}^{-1}\right)$ to minimize the effect of e-beam induced changes in the film. Samples were heated @ $5{ }^{\circ} \mathrm{C} / \mathrm{s}$ from room temperature to $180{ }^{\circ} \mathrm{C}$. The images and videos have been processed using Python scripting within the Digital Micrograph platform to increase the contrast and visibility. The chemistry of 
the as-deposited and heat-treated $\mathrm{Ge}_{4} \mathrm{Sb}_{4} \mathrm{Te}_{5}$ film was analyzed through STEM-XEDS, employing FEI G2 80-200 chemiSTEM in probe corrected mode.

The bright-field TEM images with embedded selected area electron diffraction (SAD) patterns are shown both for the as-deposited case and after heat treatment at $180{ }^{\circ} \mathrm{C}$ in Figure 1 . The BF micrograph is essentially featureless and the presence of diffuse rings in the BF-TEM image of as-deposited GST-445 suggests that the film is amorphous in nature (cf. Fig. 1a and inset therein). The presence of nucleated crystals appears darker in the BF image of the heat-treated film due to diffraction contrast (Fig. 1b). The SAD pattern corresponding to the heat-treated film at $180{ }^{\circ} \mathrm{C}$ shows the presence of rings which could be indexed to those of FCC phase. The low-dose image of the GST-445 film at room temperature recorded with a large field of view, high-speed K3 IS camera and the corresponding Python-processed image are presented in Figure 2. The recorded image along with an inset giving the FFT from a region of interest is shown in Figure 2(a); the FFT shows spots corresponding to long-range periodicity in the film. This image has been processed with Python scripting and the corresponding color-coded map is presented in Figure 2(b). Colored regions indicate crystalline domains with different orientations (depicted as color wheel as inset in Fig. 2b). These observations indicate the presence of crystals in the as-deposited GST-445 film which can be ascribed to exposure of the film to the atmosphere or quenched in nuclei during deposition. This was made possible by the detection capabilities of the K3 IS camera coupled with Python scripting. This research is supported by NSF under award DMR-1710468. The TEM studies were carried out at the Center for Integrated Nanotechnologies, an Office of Science User Facility operated for the U.S. Department of Energy (DOE) Office of Science., and in the Materials Characterization Department, Sandia National Laboratories. The authors thank Drs. Katherine Jungjohann, Matthew T. Janish and Ali Gokirmak for helpful discussions and Drs. Rongjun Wang and Sundar Ramamurthy for providing the GST material. Use of the K3 IS camera was provided courtesy of Gatan. The Sandia National Laboratories are managed and operated by National Technology and Engineering Solutions of Sandia, LLC., a wholly owned subsidiary of Honeywell International, Inc., for the U.S. DOE's NNSA under contract DE-NA-0003525. The views expressed here do not necessarily represent the views of the U.S. DOE or the U.S. Government. Los Alamos National Laboratory, an affirmative action equal opportunity employer, is managed by Triad National Security, LLC for the U.S. Department of Energy's NNSA, under contract 89233218CNA000001.
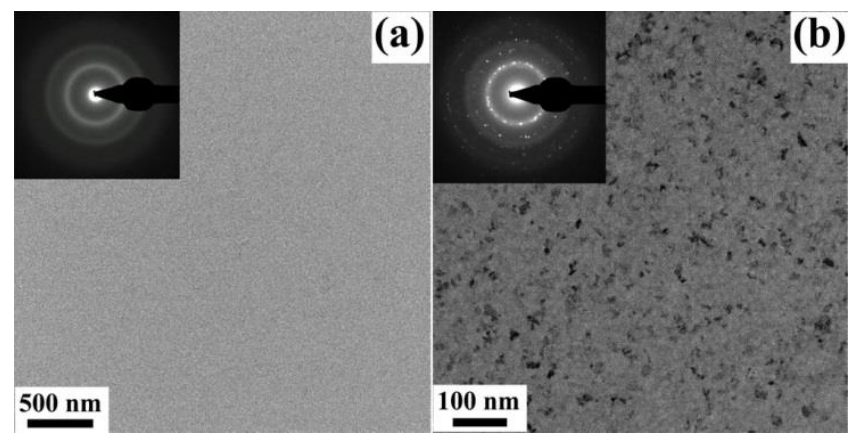

Figure 1. BF-TEM micrographs along with corresponding selected area electron diffraction patterns as insets of GST-445 film (a) in as-deposited condition and (b) heat-treated at $180{ }^{\circ} \mathrm{C}$. 


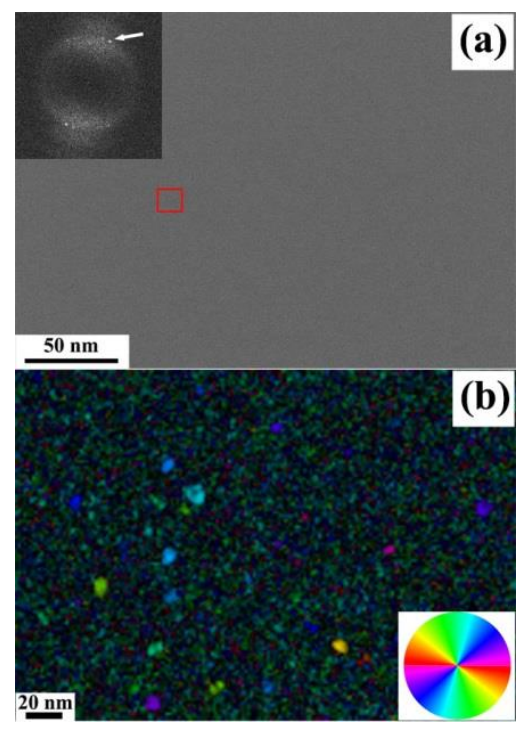

Figure 2. (a) A low electron-dose image of GST-445 film in as-deposited condition acquired by a largefield-of-view and high-speed camera along with embedded FFT from a ROI marked with red square box, (b) corresponding Python processed image with color wheel showing the orientation of the crystalline domains.

References

Ghosh, C., Singh, M.K., Watt, J., Silva, H. \& Carter, C.B. (2020). Defect Imaging and Structure Evolution in GST Films During In-situ Heating. Microscopy and Microanalysis 26(S2), 1396-1398.

Singh, M.K., Ghosh, C., Kotula, P., Watt, J., Silva, H. \& Carter, C.B. (2020a). Direct Observation of Phase Transformations in Ge-Sb-Te Materials. Microscopy and Microanalysis 26(S2), 1418-1420.

Singh, M.K., Ghosh, C., Miller, B., Kotula, P.G., Tripathi, S., Watt, J., Bakan, G., Silva, H. \& Carter, C.B. (2020b). In situ TEM study of crystallization and chemical changes in an oxidized uncapped Ge2Sb2Te5 film. J Appl Phys 128(12), 124505.

Tripathi, S., Kotula, P., Singh, M.K., Ghosh, C., Bakan, G., Silva, H. \& Carter, C.B. (2020). Role of Oxygen on Chemical Segregation in Uncapped Ge2Sb2Te5 Thin Films on Silicon Nitride. Ecs J Solid State Sc 9(5), 054007.

Wuttig, M. \& Yamada, N. (2007). Phase-change materials for rewriteable data storage. Nature materials 6(11), 824-832.

Yamada, N. (2012). Origin, secret, and application of the ideal phase-change material GeSbTe. physica status solidi (b) 249(10), 1837-1842. 